\title{
BMJ Open Canada-DONATE study protocol: a prospective national observational study of the medical management of deceased organ donors
}

\author{
Frederick D'Aragon, ${ }^{1,2}$ Sonny Dhanani, ${ }^{3}$ Francois Lamontagne, ${ }^{2,4}$ \\ Deborah J Cook, ${ }^{5,6}$ Karen Burns, ${ }^{7,8}$ Aemal Akhtar, ${ }^{6}$ Michaël Chassé, ${ }^{9}$ \\ Anne-Julie Frenette, ${ }^{10,11}$ Sean Keenan, ${ }^{12}$ Jean-Francois Lize, ${ }^{9}$ \\ Demetrios J Kutsogiannis, ${ }^{13}$ Andreas Kramer, ${ }^{14}$ Lori E Hand, ${ }^{6}$ Erika Arseneau, ${ }^{6}$ \\ Marie-Helene Masse, ${ }^{2}$ Christine Ribic, ${ }^{5,15}$ lan Ball, ${ }^{16,17}$ Andrew Baker, ${ }^{7,18}$ \\ Gordon Boyd, ${ }^{19,20}$ Bram Rochwerg, ${ }^{5,6}$ Andrew Healey, ${ }^{5}$ Steven Hanna, ${ }^{6}$ \\ Gordon H Guyatt, ${ }^{5,6}$ Maureen O Meade, ${ }^{5,6}$ for the Canadian Critical Care Trials \\ Group and the Canadian National Transplant Research Program
}

To cite: D'Aragon F, Dhanani S, Lamontagne $F$, et al. Canada-DONATE study protocol: a prospective national observational study of the medical management of deceased organ donors. BMJ Open 2017;7:e018858. doi:10.1136/ bmjopen-2017-018858

- Prepublication history and additional material for this paper are available online. To view these files, please visit the journal online (http://dx.doi. org/10.1136/bmjopen-2017018858).

Received 28 July 2017 Accepted 1 August 2017

CrossMark

For numbered affiliations see end of article.

Correspondence to Dr Frederick D'Aragon; Frederick.DAragon@ USherbrooke.ca

\begin{abstract}
Introduction Research on the management of deceased organ donors aims to improve the number and quality of transplants and recipient outcomes. In Canada, this research is challenged by regionalisation of donation services within provinces and the geographical, clinical and administrative separation of donation from transplantation services. This study aims to build a national platform for future clinical trials in donor management. Objectives are to engage collaborators at donation hospitals and organ donation organisations (ODOs) across Canada, describe current practices, evaluate the effectiveness of donation-specific interventions and assess the feasibility of future clinical trials.
\end{abstract}

Methods and analysis This ongoing prospective observational study of the medical management of deceased organ donors will enrol more than 650 consented potential donors from adult intensive care units at 33 hospital sites across Canada, each participating for 12 months. OD0s ensure enrolment of consecutive eligible participants. Research staff record detailed data about participants, therapies, organ assessments, death declaration procedures and adverse clinical exposures from the time of donation consent to organ recovery. ODOs provide reasons that organs are declined, dates and places of transplantation, and recipient age and sex. Descriptive analyses will summarise current practices. Effectiveness analyses will examine donation-specific interventions with respect to the number of transplants, using multilevel regression models to account for clustering by donor, hospitals and ODOs. Feasibility analyses will focus on acceptance of the research consent model; participation of academic and community hospitals as well as ODOs; and accessibility of recipient data.

Ethics and dissemination This study uses a waiver of research consent. Hospitals will receive reports on local practices benchmarked to (1) national practices and (2) national donor management guidelines. We will report findings to donation and transplant collaborators
Strengths and limitations of this study

This is the first Canadian prospective observational study aimed at improving medical management of organ donors.

- Prospective and consecutive enrolment of newly consented organ donors at each study site will minimise selection bias.

- Although not all provinces and territories are able to participate, including 34 hospital sites (four provinces with both community and academic hospitals) with the highest volume of deceased organ donors will increase the generalisability of the results.

- Canada-DONATE is the first national study to bridge donor and recipient data and uses a waiver of research consent.

- Due to the novelty of this study and the waived consent model a limited amount of data on corresponding recipients of enrolled organ donors is collected.

(ie, clinicians, researchers, 0D0s) and publish in peerreviewed journals.

Trial registration number NCT03114436.

\section{INTRODUCTION}

In 2010, WHO called for an international effort to increase the supply of organs for transplantation through research and education. ${ }^{1}$ Universally, the demand for organ transplantation (most commonly kidney, followed by liver, lung, heart and pancreas) exceeds organ supply, and this gap will continue to grow over the next decade. ${ }^{2}$ In Canada, more than 4000 people are registered on 
a transplant waiting list, ${ }^{3}$ of which 10 die every month awaiting transplantation.

Deceased donors provide the majority of organs for transplantation in Canada through one of two pathways. Both transpire within intensive care units (ICUs), under the care of critical care physicians and allied health professionals. Donation after a neurological determination of death (NDD) can occur after a patient with a catastrophic brain injury fulfils diagnostic criteria for brain death. Donation after a circulatory determination (DCD) of death can occur following a planned, palliative withdrawal of life-sustaining technologies. ICU management strategies aim to normalise physiological parameters in the organ donor to preserve or augment organ function for transplantation. Specifically, clinicians provide vasoactive medications, lung-protective mechanical ventilation, hormone supplementation, nutrition, electrolyte and glucose control, systemic anticoagulation as well as advanced haemodynamic monitoring and bronchoscopy. ${ }^{4-6}$

In 2015, the Society of Critical Care Medicine set out to develop evidence-based guidelines in donor management, but published, instead, consensus-based guidelines, citing that '...the available literature overwhelmingly comprised observational studies and retrospective case series, representing low-quality evidence, with a notable scarcity of randomised controlled trials'. ${ }^{6}$ Because of the paucity of experimental research in this field, the benefits (or harms) of ICU strategies for preserving organ function and improving recipient outcomes are uncertain. ${ }^{6}$ Observational studies suggest, however, that after consent for organ donation, up to $20 \%$ of organs may lose transplant potential due to suboptimal medical management. ${ }^{7-9}$ Clinical research to elucidate the effects of various donor management strategies is likely to enhance the number and quality of transplanted organs, potentially saving lives and improving quality of life. ${ }^{10} 11$

Clinical trials of novel interventions in organ donation are especially scarce, ${ }^{12}$ largely because researchers in this field face unique challenges. Administration of study interventions in organ donors with the need to measure outcomes among organ recipients is scientifically complicated and also fraught with practical difficulties. The regionalisation of health services across Canadian provinces is one example. In Canada, hundreds of ICUs are responsible for the identification and referral of potential donors to provincial organ donation organisations (ODOs). With varied levels of resources, ODOs coordinate donation services across each province. Donated organs frequently cross provincial borders and the subsequent medical care of transplant recipients falls under the domain of 72 transplant programmes in Canada. Thus, the flow of organs is complex, regionalised and involves various distinct institutions across the country. Different provincial privacy laws further complicate procedures for research consent and data acquisition. Linking donor data to recipient data is complex in this milieu.
Also challenging is the traditional separation of donation services from transplantation services, both clinically and administratively. Historically, this served to protect prospective organ donors from potential conflict of interest of clinicians, and also to honour the privacy of donors, recipients and their families. A consequence has been the creation of detached programmes for clinical care and for clinical research where, in fact, closer collaboration between donation and transplantation communities would advance the field.

The first objective of this research programme is to build a national platform to support randomised clinical trials in deceased organ donation. This will require several important steps: recruiting hospitals, building their research capacity, learning about current clinical practices, streamlining procedures for enrolment and complex data capture and integration, and forging strong research collaborations with ODOs and transplant programmes across the country. Other objectives of the study are to fill important knowledge gaps. Few studies have described the ICU management of deceased donors. ${ }^{13}$ This study will describe the variability of medical practice in deceased donor care across Canada, highlighting regional differences, and will investigate the therapeutic effectiveness of various donor-specific ICU interventions.

\section{METHODS AND ANALYSIS \\ Study design}

This protocol describes a multicentre, prospective observational cohort study evaluating the ICU management of consented organ donors in Canada. The study is currently under way (NCT03114436), and builds on the completed, city-wide, Hamilton-DONATE Pilot Study ( $\mathrm{n}=68$; NCT02902783), undertaken from August 2015 to August 2016 in Hamilton, Ontario, Canada. The coordinating centre is CLARITY Research at McMaster University, Hamilton, Canada.

\section{Study setting}

Thirty-four active deceased donation hospital sites in Canada (many with multiple ICUs) are currently participating, with representation from the provinces of British Columbia, Alberta, Ontario and Quebec (table 1).

Two hospitals in Saskatchewan and Nova Scotia are also planning to participate.

\section{Eligibility criteria}

All potential organ donors admitted to participating adult ICUs (including coronary care units with intensive care services), and for whom a legal substitute decision maker has provided consent for deceased donation, are eligible for this study.

Potential organ donors who are not typically managed in an adult ICU in Canada are not included in this study. These include neonates and children managed in specialised paediatric ICUs; non-critically ill patients who 
Table 1 Active hospital sites

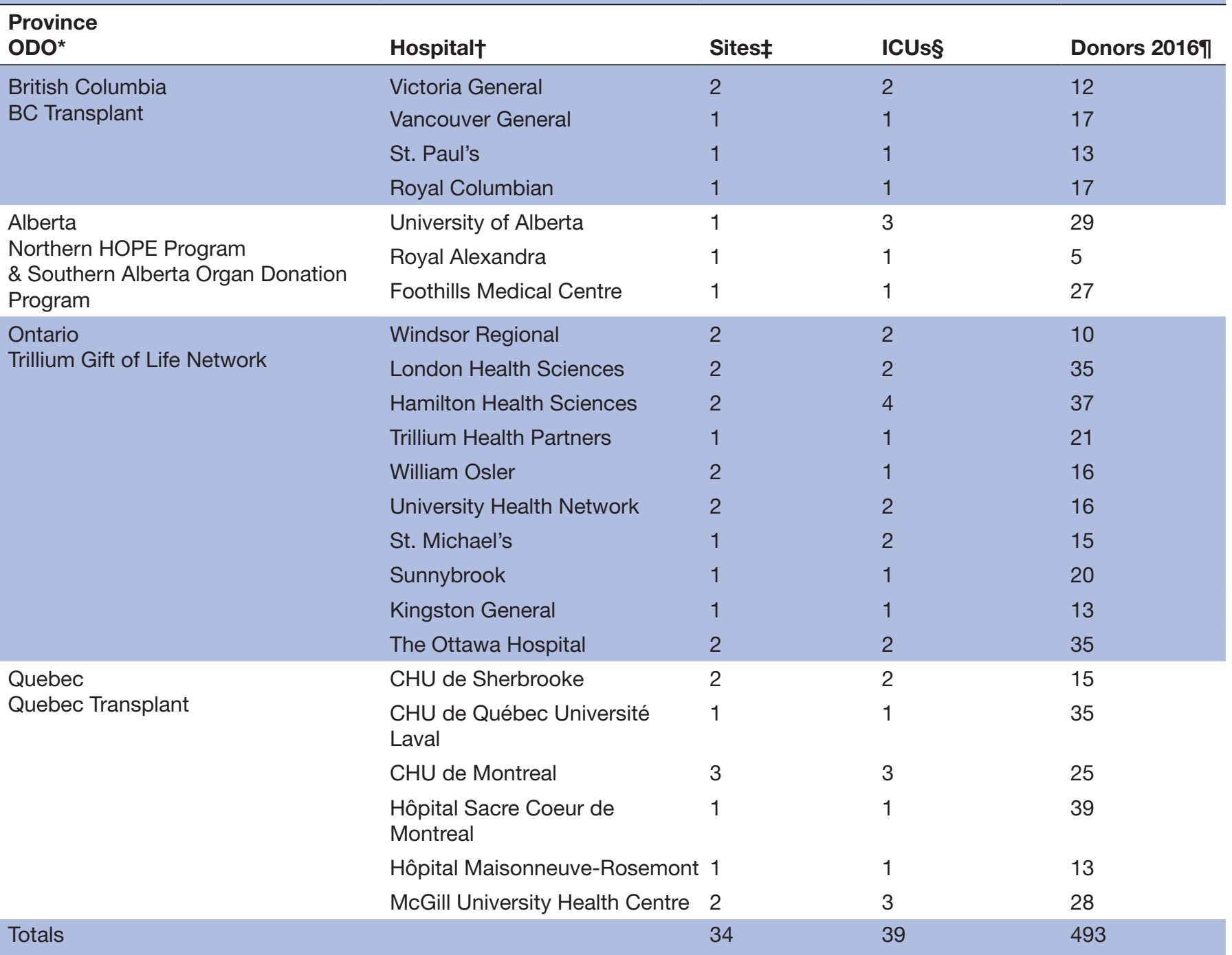

${ }^{*}$ ODOs are provincial regulatory bodies responsible for the oversight of all organ donations within their regional boundaries.

†Each hospital refers to the local organisation and may have more than one physical hospital site.

$\ddagger$ Site numbers refer to the number of physical locations contained within each local organisation (hospital).

$\S$ This number refers to the number of ICUs across all physical locations within each local organisation (hospital).

१The combined number of actual donors in 2016 at each hospital. This is an estimate of the number of actual donors that will be recruited within 1 year.

ICU, intensive care unit; ODO, organ donation organisation.

undergo donation after medical assistance in dying and victims of cardiac arrest who fail to regain a sustained, perfusing cardiac rhythm.

\section{Identification of consented organ donors}

Procedures for identifying consented potential donors require the support of ODOs and thus vary across the provinces according to available technology and human resources. In each province, ODO personnel solicit and register consent for deceased donation and promptly notify central study personnel. Wherever possible, this notification is automated. For example, in Ontario the ODO sends an automatic email alert to the central study manager when a newly consented donor is registered. In Quebec, the ODO personnel notify ICU research staff and coordinating research staff of every newly consented organ donor in the province. To safeguard against any lapse in these systems, ICU research staff also screen patients daily during regular working hours for potential organ donors.

\section{Enrolment}

ICU research staff record the ODO referral number, assign each consented donor a unique study identification number and register the patient into the electronic study database.

\section{Data sources}

Clinical data relevant to this study are similar to data collected by ODOs (see online supplementary appendix 1 ); however, the study data are generally more detailed. Data sources include direct donor observation, electronic 
and paper hospital charts, and regional electronic records. ODOs provide additional data to the coordinating centre on a regular basis, as outlined below.

\section{Time frame for data collection}

Retrospective data includes previous medical history and details of the illness precipitating ICU admission. To the extent that it is possible to collect the remaining data prospectively, ICU research staff will do so up to the time of surgery for organ recovery. They develop local strategies to retain time-sensitive data from evenings and weekends, including data that is typically poorly documented in hospital records (eg, bedside echocardiography) or requires physician interpretation (eg, diagnosing ventilator-associated pneumonia). For study participants who are ultimately unable to donate, we collect data up to the time of this decision.

\section{Data types}

All consented donors

ICU research staff record extensive data related to donor management which are summarised in table 2 and online supplementary appendix 2.

\section{Data management and validation}

Case report forms (CRFs) were refined from the preceding pilot study (see online supplementary appendix 1). Detailed instruction regarding CRF completion is available at start-up visits at each site, in an accompanying data manual and in a 'Frequently Asked Questions' page on the study microsite (http://donate.ccetg.ca).

ICU research staff record data on electronic CRFs for encrypted transmission to the coordinating centre using the internet-based iDataFax System (DF/Net Research). Automated data verification programmed within the active iDataFax system alerts these research staff to potential errors (extreme or missing values). Research personnel at the coordinating centre review incoming data for accuracy and completeness, generating data queries for ICU research staff to resolve before a CRF is designated as 'complete'.

\section{Outcome measurements}

Outcome measurements in this study relate to describing practice patterns, analysing the effectiveness of various interventions and assessing the feasibility of future clinical trials.

Outcomes relevant to the description of practice patterns include, but are not limited to: (1) adherence to the recommendations in current national guidelines for the management of deceased organ donors (eg, administration of systemic corticosteroids, thyroid hormone and vasopressin; glycaemic control with insulin infusion; transfusion of blood products at specified thresholds; application of lung-protective ventilation modalities), ${ }^{5}$ (2) the use of specific technologies in organ donor management and evaluation (eg, bronchoscopy, radiocontrast and biopsy use in organ assessments, ancillary tests for brain death), (3) clinical diagnosis of catecholamine storm or
Table 2 Overview of data collection from consented deceased organ donors

\begin{tabular}{|c|c|}
\hline Data type & Data capture includes \\
\hline \multirow[t]{4}{*}{ Monitoring devices } & Arterial catheter \\
\hline & Central venous catheter \\
\hline & Pulmonary artery catheter \\
\hline & Echocardiography \\
\hline \multirow{6}{*}{$\begin{array}{l}\text { Advanced organ-sustaining } \\
\text { therapies }\end{array}$} & Mechanical ventilation \\
\hline & Vasopressors and inotropes \\
\hline & $\begin{array}{l}\text { Cardiopulmonary } \\
\text { resuscitation }\end{array}$ \\
\hline & Renal replacement therapy \\
\hline & Fluid resuscitation \\
\hline & Enteral/parenteral nutrition \\
\hline \multirow[t]{4}{*}{ Prophylactic medications } & Gastrointestinal prophylaxis \\
\hline & Thromboprophylaxis \\
\hline & Pneumonia prophylaxis \\
\hline & Glycaemic control \\
\hline \multirow[t]{6}{*}{ Donation-specific therapies } & Glucocorticosteroids \\
\hline & Thyroid hormone \\
\hline & Vasopressin infusions \\
\hline & DDAVP administration \\
\hline & Therapeutic heparin \\
\hline & Antimicrobial therapy \\
\hline \multirow[t]{5}{*}{$\begin{array}{l}\text { Potentially adverse ICU } \\
\text { exposures }\end{array}$} & $\begin{array}{l}\text { Oxygen desaturation }\left(\mathrm{SpO}_{2}\right. \\
<88 \% \text { for } \geq 15 \mathrm{~min})\end{array}$ \\
\hline & $\begin{array}{l}\text { Haemodynamic instability } \\
\text { (mean arterial pressure } \\
<65 \mathrm{~mm} \mathrm{Hg} \text { for } \geq 15 \text { min or } \\
\text { new vasopressors initiated } \\
\text { for treatment of hypotension) }\end{array}$ \\
\hline & $\begin{array}{l}\text { Cardiac arrhythmias requiring } \\
\text { chemical or electrical } \\
\text { cardioversion }\end{array}$ \\
\hline & $\begin{array}{l}\text { Active bleeding requiring } \\
\text { blood transfusion }\end{array}$ \\
\hline & $\begin{array}{l}\text { Extremes of body } \\
\text { temperature }\left(<36^{\circ} \mathrm{C}\right. \\
\left.\text { or }>38.5^{\circ} \mathrm{C}\right)\end{array}$ \\
\hline \multirow{3}{*}{$\begin{array}{l}\text { Neurological death } \\
\text { declaration }\end{array}$} & Time of death \\
\hline & $\begin{array}{l}\text { Details related to } \\
\text { apnoea test }\end{array}$ \\
\hline & Ancillary tests \\
\hline \multirow[t]{3}{*}{$\begin{array}{l}\text { Cardiocirculatory death } \\
\text { declaration }\end{array}$} & $\begin{array}{l}\text { Sequence of advanced } \\
\text { organ-sustaining therapy } \\
\text { withdrawal }\end{array}$ \\
\hline & $\begin{array}{l}\text { Changes in dose of palliative } \\
\text { medications }\end{array}$ \\
\hline & $\begin{array}{l}\text { Evolution of vital signs } \\
\text { after withdrawal of organ- } \\
\text { sustaining therapy }\end{array}$ \\
\hline
\end{tabular}

Continued 
Table 2 Continued

\begin{tabular}{ll}
\hline Data type & Data capture includes \\
\hline Organ suitability assessments & Blood tests \\
& Chest radiography \\
& Bronchoscopy \\
& Body imaging \\
& Echocardiography \\
& Coronary angiography \\
& Microbiological cultures \\
& Biopsies \\
& Time and rationale for organ \\
decline & Age \\
Transplant recipients & Sex \\
& Panel reactive antigen \\
& Transplant programme \\
& Transplant date
\end{tabular}

ICU, intensive care unit.

ventilator-associated pneumonia, (4) meeting specific study criteria for hypotension, desaturation and cardiac arrhythmias.

We will capture outcomes relevant to measuring the effectiveness of various interventions including: clinical stabilisation, organ recovery, multiple organ recovery, organ transplantation and the reasons for which specific organs were declined for transplantation.

Additionally we will record outcomes relevant to advancing clinical research in this field including: the number of hospitals unable to participate in this study due to the waiver of research consent, missed eligible participants, withdrawal of eligible participants, acquisition of unambiguous data on the timing and rationale for organ declines, and acquisition of complete data on transplant recipients.

\section{Analyses}

A PhD-level faculty biostatistician with expertise in large observational datasets and multilevel regression modelling will oversee these analyses. All regression models outlined in the Descriptive analyses, Effectiveness analyses and Feasibility analyses subsections will include assessments for collinearity, interactions and model fit. Study inferences about statistical comparisons will apply a nominal $p$ value of 0.05 . The multiple imputation method with sensitivity analyses described by Molenberghs and Kenward will be used to address missing data where appropriate. ${ }^{14}$ Mixed effects random forest models will be used if parametric assumptions are problematic. ${ }^{15}$ Guidelines outlined by the Strengthening the Reporting of Observational Studies in Epidemiology statement will be followed when reporting study findings. ${ }^{16}$

\section{Descriptive analyses}

Donor data and practice patterns will be analysed using descriptive statistics, reporting means (SD), medians (first quartile, third quartile) or proportions, as appropriate. The unit of these analyses is the consented organ donor. We will analyse the proportion of all consented donors who receive specific interventions (eg, corticosteroid therapy, various components of a lung-protective ventilation strategy or ancillary test of brain death). For selected interventions, we will also assess for variables that predict or are associated with their use (eg, age or the specified target organs from a particular donor). For these analyses, we will use multilevel regression models (to account for clustering within provinces, cities, hospitals, ICUs or by donor or organ type, as appropriate). We will similarly analyse the rate of adherence to discrete components of current national guidelines.

\section{Effectiveness analyses}

To assess the benefits (or harms) of specific interventions, we will also use multilevel regression models. However, the unit of these analyses will be the potential organs (rather than the consented donors). Dependent variables will include (1) the number of study participants from whom organs are actually surgically recovered, (2) the number of specific organs that are surgically recovered (where applicable; eg, lungs, in the case of lung-protective ventilation), (3) the mean number of all organs recovered per study participant and (4) the mean number of organs transplanted per donor. We will assess the impact of key independent variables (eg, presence or absence of lung-protective ventilation) first with unadjusted comparisons. Then, we will adjust for other variables known to influence transplantability (eg, age, donor type and donor management time). These analyses will only apply for interventions where practices are not highly consistent across sites.

\section{Feasibility analyses}

To assess feasibility of future clinical trials, we will analyse the time to finalise regulatory approvals at each hospital and ODO from the time of first application; and the ability of ODOs to inform the coordinating centre of all consented organ donors in real time. We will analyse the completeness (vs missingness) of ODO data related to organ declines and transplant recipients. We will also describe the variability in time to regulatory approvals and completeness of ODO data.

Future clinical trials evaluating the impact of ICU interventions on the number of organs transplanted will require accurate determinations of (1) which organs had potential for donation at the time of consent, (2) which organs were declined by transplant programmes (and the timing and justification for their decline) and (3) which organs were ultimately transplanted. We will assess the completeness of these data as recorded on the CRFs and as received from ODOs, and will adjudicate these determinations in duplicate. 


\section{Sample size}

The anticipated sample size for this observational study stems from the goal of meeting all objectives of the study. In creating a national platform for future clinical trials, we sought to include (1) all donation hospitals in Canada with active critical care research infrastructure, (2) the highest-activity donation hospitals in each province (eg, more than 10 donors per year, regardless of existing research infrastructure) and (3) additional hospitals that expressed a strong interest in collaborating. This approach resulted in the inclusion of both academic and community hospitals, which will enhance the generalisability of descriptive study findings. We also sought to achieve as many consented donors as possible to maximise the precision of regression analyses testing the effectiveness of various ICU interventions.

Based on 2016 data from ODOs there were 493 deceased donors at the 34 participating hospital sites across Canada. Based on the rate of conversion of consented to actual donors derived from the Hamilton Pilot Study (74\%), we expect to enrol approximately 666 consented deceased organ donors. Approximately 500 of these consented donors will provide organs for transplantation and, assuming a rate of 3.2 organs per donor (from our Hamilton pilot study), this will amount to 1600 organs for transplantation.

The anticipated sample of 666 consented potential organ donors will provide ample power for all of the analyses that we have planned: the descriptive analyses, the analyses of the determinants of use of various interventions and the comparative effectiveness analyses of different interventions. For instance, for the comparative effectiveness analyses, where the unit of analysis is the potential organ, this sample will afford a power of greater than $90 \%$ to detect a difference of 0.25 in the mean organ recovery rate (per donor) observed between two interventions (eg, lung-protective ventilation vs conventional ventilation) and a power of greater than $99 \%$ to detect a difference of 0.5 , assuming a SD 1.5 organs recovered per donor, ${ }^{3}$ and a nominal $p$ value of 0.05 . The power of these analyses will also depend on the degree of clustering. For regressions assessing predictors of specific practices, if we follow a conventional and conservative rule of thumb that requires at least 10 outcomes of interest for each predictor included in the multivariable model, ${ }^{17}$ we could include up to 20 variables in some regression models, far more than we anticipate.

\section{Timelines}

Regulatory reviews at hospital research ethics boards and ODO privacy offices started in Ontario and fanned out to Quebec and subsequent provinces in a stepwise fashion. Similarly, participating hospitals have been launching the study over a period of several months. Of 34 hospital sites, 33 are currently enrolling consented donors into the study, and the final participating hospital is likely to launch in September 2017. Therefore, the anticipated timeline for enrolment is April 2016 to September 2018, a period of 2.5 years.

\section{ETHICS}

This study has been approved by the Research Ethics Board of each participating hospital, the Privacy Office of each participating ODO and provincial privacy offices when necessary.

The key ethical consideration relevant to this proposal is the waiver of research consent from both NDD and DCD organ donors and from transplant recipients for whom ODOs provide minimal data. To this end, detailed documents separate from this study protocol and specific to each province provide the justification for a waiver of research consent (see online supplementary appendix 3). The framework for these documents includes consideration of the fundamental principles of ethics (autonomy, justice, equity, beneficence and non-maleficence), medical ethics (in accordance with the Second Tri-Council Policy Statement (TCPS(2))), law (national and provincial health information privacy laws), the scientific method (with the aims of minimising bias and maximising generalisability) as well as relevant precedents.

With respect to organ donors, a waiver of research consent meets criteria specified by the TCPS(2) (see online supplementary appendix 3). The study mandates no experimental tests, procedures, clinical or diagnostic interventions and therefore involves minimal risk. De-identified data is collected from routine clinical investigations. Meanwhile, a requirement for consent would introduce bias into the recruited study population versus the eligible population, and thereby increase the risk of bias of study findings. In the unlikely situation that families consent to organ donation but actively decline participation in this study, their reasons will be clearly documented.

With respect to transplant recipients, obtaining consent for observational data presents a unique challenge because: (1) transplant programmes are rarely located in organ donation hospitals; (2) organs from one donor may be dispersed to different hospitals across Canada (and beyond); (3) transplant recipients are selected within hours of organ recovery and (4) donation and transplant programmes are effectively distinct to avoid potential conflicts of interest.

\section{DISSEMINATION}

Investigators have presented the vision of this research programme along with the study protocol to administrative and clinical leaders of the provincial ODOs, specific stakeholder physicians and administrators within participating hospitals and to members of the Canadian Critical Care Trials Group, the Canadian National Transplant Research Program and the Scientific Advisory Committee of the Canadian Blood Services, which provides national oversight to donation and transplantation services in Canada. Collaborators are updated through quarterly bulletins describing current progress of the study locally and nationally. 
When the study is completed, we will collaborate with local Donation Specialist Physicians to present the findings at each participating hospital to: (1) share the overall results, (2) compare practices and outcomes across centres and (3) reinforce the role of local hospital champions (Donation Specialist Physicians) and ODOs. Each hospital will receive an executive summary of local practices benchmarked to national norms and to national guidelines. Provincial ODOs and transplant programmes will receive similar (aggregate) summaries. Study results will be presented at scientific meetings of the Canadian Critical Care Trials Group and the Canadian National Transplant Research Program, and published in peer-reviewed journals.

\section{CONCLUSION}

The optimal medical management of deceased donors is undefined because scientific evidence is lacking. This multicentre observational study will have broad impact to address current knowledge gaps and to advance this research field.

In terms of new knowledge, this study will describe for the first time the medical practices in deceased donor care across Canada, highlighting regional differences, and will investigate the therapeutic effectiveness of various donor-specific ICU interventions in the Canadian healthcare system. At the hospital level, the dissemination of results will provide support for best practices to be guided by current consensus guidelines and future evidencebased guidelines. For ODOs, this study will identify practices for which educational initiatives are required, and will serve as a unique source of information for quality improvement initiatives. At the system level, the study will generate national normative practices and help to elucidate determinants of success for centres across Canada.

The organ donation research infrastructure developed in each centre will increase the feasibility of future clinical trials on deceased donor management. Partnering in research with ODOs is pivotal to this work and will continue to enhance patient enrolment, achieve donor data needs and allow a research link between donor and recipient data. This national cohort study is an important step towards a national research programme on deceased donor management.

\footnotetext{
Author affiliations

${ }^{1}$ Department of Anesthesiology, Universite de Sherbrooke, Sherbrooke, Quebec, Canada

${ }^{2}$ Centre de Recherche du Centre Hospitalier Universitaire de Sherbrooke,

Sherbrooke, Quebec, Canada

${ }^{3}$ Division of Critical Care, Department of Pediatrics, Children's Hospital of Eastern Ontario, University of Ottawa, Ottawa, Ontario, Canada

${ }^{4}$ Department of Medicine, Universite de Sherbrooke, Sherbrooke, Ontario, Canada

${ }^{5}$ Department of Medicine, McMaster University, Hamilton, Ontario, Canada

${ }^{6}$ Department of Health Evidence and Impact, McMaster University, Hamilton,

Ontario, Canada

${ }^{7}$ Interdepartmental Division of Critical Care, University of Toronto, Toronto, Ontario, Canada

${ }^{8}$ Li Ka Shing Knowledge Institute, St. Michael's Hospital, Toronto, Ontario, Canada
}

${ }^{9}$ Department of Medicine (Critical Care), Centre Hospitalier de l'Universite de Montreal, Montreal, Quebec, Canada

${ }^{10}$ Pharmacy Faculty, Universite de Montreal, Montreal, Quebec, Canada

${ }^{11}$ Hopital Sacre-Coeur de Montreal, Montreal, Quebec, Canada

${ }^{12}$ Department of Critical Care, Royal Columbian Hospital, New Westminster, British Columbia, Canada

${ }^{13}$ Department of Critical Care Medicine, University of Alberta, Edmonton, Alberta, Canada

${ }^{14}$ Department of Critical Care Medicine, University of Calgary, Calgary, Alberta, Canada

${ }^{15}$ Division of Nephrology, St. Joseph's Healthcare, Hamilton, Ontario, Canada

${ }^{16}$ Department of Medicine, Western University, London, Ontario, Canada

${ }^{17}$ Department of Epidemiology and Biostatistics, Western University, London, Ontario, Canada

${ }^{18}$ Departments of Anaesthesia and Surgery, University of Toronto, Toronto, Ontario, Canada

${ }^{19}$ Department of Medicine (Neurology), Queen's University, Kingston, Ontario, Canada

${ }^{20}$ Department of Critical Care Medicine, Queen's University, Kingston, Ontario, Canada

Collaborators Canadian Critical Care Trials Group, the Canadian National Transplant Research Program, Canadian Blood Services, British Columbia Transplant, the Northern Alberta HOPE Program, the Southern Alberta Organ Donation Program, the Trillium Gift of Life Network of Ontario and Transplant Quebec.

Contributors All authors made material contributions to this manuscript according to the rules of authorship of ICMJE. Specifically, the contributions of each author are as follows: FD, SD, DJC, A-JF, GHG, FL, MOM: conception of the design, draft and revised the manuscript, and approved the final version to be published; $A A, E A, A B$, IB, GB, KB, MC, LEH, SH, AH, SK, AK, DJK, J-FL, M-HM, CR, BR: reviewed the design, draft and revised the manuscript, and approved the final version to be published.

Funding This work is supported by: the Hamilton Health Sciences RFA Strategic Initiatives Program; the Hamilton Health Sciences New Investigator Fund; a Canadian Blood Services Kenneth J. Fyke award; the Canadian Institutes for Health Research (FRN94829) and the Fond de Recherches de la Fondation des Anesthesiologistes du Quebec.

Competing interests None declared.

Ethics approval Hamilton Integrated Research Ethics Board.

Provenance and peer review Not commissioned; peer reviewed for ethical and funding approval prior to submission.

Open Access This is an Open Access article distributed in accordance with the Creative Commons Attribution Non Commercial (CC BY-NC 4.0) license, which permits others to distribute, remix, adapt, build upon this work non-commercially, and license their derivative works on different terms, provided the original work is properly cited and the use is non-commercial. See: http://creativecommons.org/ licenses/by-nc/4.0/

(c) Article author(s) (or their employer(s) unless otherwise stated in the text of the article) 2017. All rights reserved. No commercial use is permitted unless otherwise expressly granted.

\section{REFERENCES}

1. Shemie SD, Hornby L, Baker A, et al. International guideline development for the determination of death. Intensive Care Med 2014;40:788-97.

2. Canadian Institute for Health Information. Deceased organ donor potential in Canada. Ottawa, ON: Canadian Institute for Health Information, 2014.

3. Canadian Institute for Health Information. Canadian organ replacement register annual report: treatment of end-stage organ failure in Canada, 2004-2013. Ottawa, ON: Canadian Institute for Health Information, 2015.

4. Shemie SD, Baker AJ, Knoll G, et al. National recommendations for donation after circulatory death in Canada. Can Med Assoc J 2006;175:S1-24.

5. Shemie SD, Ross H, Pagliarello J, et al. Organ donor management in Canada: recommendations of the forum on medical management to optimize donor organ potential. CMAJ 2006;174:S13-30. 
6. Kotloff RM, Blosser S, Fulda GJ, et al. Management of the potential organ donor in the ICU: Society of Critical Care Medicine/ American College of Chest Physicians/Association of Organ Procurement Organizations Consensus Statement. Crit Care Med 2015;43:1291-325.

7. Mascia L, Mastromauro I, Viberti S, et al. Management to optimize organ procurement in brain dead donors. Minerva Anestesiol 2009; $75: 125-33$.

8. Shah VR. Aggressive management of multiorgan donor. Transplant Proc 2008;40:1087-90.

9. Dikdan GS, Mora-Esteves C, Koneru B. Review of randomized clinical trials of donor management and organ preservation in deceased donors: opportunities and issues. Transplantation 2012;94:425-41.

10. Floerchinger B, Oberhuber R, Tullius SG. Effects of brain death on organ quality and transplant outcome. Transplant Rev 2012;26:54-9.
11. Bos EM, Leuvenink HG, van Goor $\mathrm{H}$, et al. Kidney grafts from brain dead donors: Inferior quality or opportunity for improvement? Kidney Int 2007;72:797-805.

12. Greer DM, Valenza F, Citerio G. Improving donor management and transplantation success: more research is needed. Intensive Care Med 2015;41:537-40.

13. Dhanani S, Shemie SD. Advancing the science of organ donor management. Crit Care 2014;18:612-4.

14. Molenberghs G, Kenward M. Missing data in clinical trials. Hoboken, New Jersey: John Wiley \& Sons, 2007.

15. Hajjem A, Bellavance F, Larocque D. Mixed-effects random forest for clustered data. J Stat Comput Simul 2014;84:1313-28.

16. Elm Ev, Altman DG, Egger M, et al. Strengthening the reporting of observational studies in epidemiology (STROBE) statement: guidelines for reporting observational studies. BMJ 2007;335:806-8.

17. Vittinghoff $E$, McCulloch $C E$. Relaxing the rule of ten events per variable in logistic and Cox regression. Am J Epidemiol 2007;165:710-8. 